\title{
DTi2Vec: Drug-target interaction prediction using network embedding and ensemble learning
}

\author{
Maha A. Thafar ${ }^{1,2}$, Rawan S. Olayan³, Somayah Albaradei ', ${ }^{1,4}$, Vladimir B. Bajic ${ }^{1}$, Takashi Gojobori', \\ Magbubah Essack ${ }^{1^{*}}$ and Xin Gao ${ }^{1 *}$
}

\begin{abstract}
Drug-target interaction (DTI) prediction is a crucial step in drug discovery and repositioning as it reduces experimental validation costs if done right. Thus, developing in-silico methods to predict potential DTI has become a competitive research niche, with one of its main focuses being improving the prediction accuracy. Using machine learning (ML) models for this task, specifically network-based approaches, is effective and has shown great advantages over the other computational methods. However, ML model development involves upstream hand-crafted feature extraction and other processes that impact prediction accuracy. Thus, network-based representation learning techniques that provide automated feature extraction combined with traditional ML classifiers dealing with downstream link prediction tasks may be better-suited paradigms. Here, we present such a method, DTi2Vec, which identifies DTIs using network representation learning and ensemble learning techniques. DTi2Vec constructs the heterogeneous network, and then it automatically generates features for each drug and target using the nodes embedding technique. DTi2Vec demonstrated its ability in drug-target link prediction compared to several state-of-the-art network-based methods, using four benchmark datasets and large-scale data compiled from DrugBank. DTi2Vec showed a statistically significant increase in the prediction performances in terms of AUPR. We verified the "novel" predicted DTIs using several databases and scientific literature. DTi2Vec is a simple yet effective method that provides high DTI prediction performance while being scalable and efficient in computation, translating into a powerful drug repositioning tool.
\end{abstract}

Keywords: Drug repositioning, Drug-target interaction, Heterogeneous network, Network embedding, Random walk, Link prediction, Representation learning, Ensemble learning, Cheminformatics

\section{Introduction}

Identifying novel drug-target interactions (DTIs) is crucial to various biomedical and polypharmacology applications, such as drug discovery, drug repositioning [1], drug resistance, and side-effect prediction [2]. However, high experimental validation costs plague its success.

\footnotetext{
*Correspondence: magbubah.essack@kaust.edu.sa; xin.gao@kaust.edu.sa ${ }^{1}$ Computer, Electrical and Mathematical Sciences and Engineering Division (CEMSE), Computational Bioscience Research Center, Computer (CBRC), King Abdullah University of Science and Technology (KAUST), Thuwal, Kingdom of Saudi Arabia
}

Full list of author information is available at the end of the article
Thus, before pursuing costly practical endeavors, more research efforts are now directed towards computationally predicting the more feasible DTIs first. Identifying these feasible DTIs can be ascertained in different ways (i.e., using various DTIs prediction tasks) such as via (1) determining if a drug interacts with the target or not (i.e., binary classification) $[3,4],(2)$ predicting the drugs' binding affinity towards the target protein (i.e., regression) $[5$, 6], or (3) predicting if the drug inhibits or enhances the reaction that occurs in the cell when the target is bound [7].

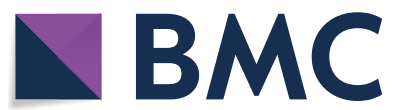

(c) The Author(s) 2021. Open Access This article is licensed under a Creative Commons Attribution 4.0 International License, which permits use, sharing, adaptation, distribution and reproduction in any medium or format, as long as you give appropriate credit to the original author(s) and the source, provide a link to the Creative Commons licence, and indicate if changes were made. The images or other third party material in this article are included in the article's Creative Commons licence, unless indicated otherwise in a credit line to the material. If material is not included in the article's Creative Commons licence and your intended use is not permitted by statutory regulation or exceeds the permitted use, you will need to obtain permission directly from the copyright holder. To view a copy of this licence, visit http://creativecommons.org/licenses/by/4.0/. The Creative Commons Public Domain Dedication waiver (http://creativeco mmons.org/publicdomain/zero/1.0/) applies to the data made available in this article, unless otherwise stated in a credit line to the data. 
Several methods have been developed based on these various DTI prediction tasks. According to recent reviews focused on DTIs prediction methods [4, 8-12], we can classify such prediction methods into three main categories, namely: docking-based approaches [13-15], ligand-based approaches [16, 17], and chemogenomicbased approaches $[4,8]$. Of these approaches, the go-to approach is chemogenomic-based $[10,18]$. The chemogenomic-based approaches overcome the limitations of docking-based approaches (3D structure information for many target proteins are unavailable) and ligand-based approaches (the number of known ligands is limited or few), utilizing the chemical and genomic information of drugs and the target proteins instead. The chemogenomic-based approaches incorporate DTI prediction models based on network-, machine learning (ML)-, and deep learning (DL)-based methods. The ML-based methods use a feature-based approach that represents each drug-target pair by hand-crafted extracted features/feature vectors (FV) $[8,10,19]$, or a similaritybased approach built based on the "guilt-by-association" hypothesis that similar drugs tend to interact with similar target proteins and vice versa [20,21]. DL-based methods are a special type of ML that can learn representations of data with multiple abstraction levels and proved its efficiency in the biomedical domain [22, 23], including DTIs prediction [24].

This article focuses on the network-based methods; specifically, the network embedding-based methods, since our method, DTi2Vec, and the compared stateof-the-art methods belong to this category. Networkbased methods [9] have been developed by formulating the prediction of DTIs as a link prediction problem in a heterogeneous graph where the goal is to uncover a novel interaction or link between drugs and targets. The network-based methods include traditional graph-based methods [9, 25-29] and network embedding methods. Traditional network-based methods predict DTIs through bipartite local model (BLM) [30, 31], networkbased inference (NBI) model [32,33], path score model (PSM) $[27,28,34]$, and a special type of network-based method that are knowledge graph-based [35]. These traditional network-based methods proved their efficiency in solving DTIs link prediction. For example, DDR [27], a PSM-based method, proved its efficiency in predicting novel DTIs. It constructs a heterogeneous graph consisting of known DTIs, multiple drug-drug similarities, and multiple target-target similarities. Similarity selection and similarity integration algorithms select a subset of the similarities then fuse them using a nonlinear function (SNF) [44]. The path score for different path categories is generated as features, and then these features are fed into the RF classifier for prediction. However, such methods suffer from some limitations. They require a lot of network analysis and can only leverage from a specific subset of statistics and ML techniques due to challenges associated with handling the sparsity and high dimensionality of heterogeneous DTI networks. Thus, network-embedding methods [36-39] have emerged as a new efficient and promising paradigm to address traditional network analysis limitations. Network embedding converts the graph into low-dimensional space while preserving the network's structural and topological information maximally. Compressed yet informative FV represents the DTIs in the embedding space, and downstream link prediction tasks can be done using ML or DL classifiers. Categories of network embedding-based methods include the random walk-based method [40], matrix factorization (MF) based methods [41], and neural network (NN) based methods [42]. DNILMF [43] (Dual Network Integrated Logistic Matrix Factorization) is an MF-based method that integrates different similarity measures for both drugs and targets by applying a nonlinear similarity fusion technique based on the similarity network fusion method (SNF) [44]. Then it used this final combined measure to predict DTIs based on their graph neighbors. NRLMF [45] (Neighborhood Regularized Logistic Matrix Factorization) is another MF state-of-the-art method that integrated logistic MF with neighborhood regularization for DTI prediction by modeling the DTI probability for each drug-target pair using logistic MF. Next, it extracted drug-specific and target-specific latent feature vectors that represented drug and target properties. It also includes the local structure of DTIs to improve the interaction and use the drug similarities and target similarities in terms of nearest neighbors to eliminate the noise from using all similar neighbors. TriModel [37] (a very recent network-based method) utilizes a knowledge graph (KG) constructed from integrating different information sources to generate KG embeddings for entities (i.e., nodes) and relations (i.e., edges). TriModel predicted novel DTIs based on their interaction scores calculated using trained tensor factorization applied on the knowledge graph embeddings. The last state-of-the-art method worth mentioning is DTiGEMS+[28], a path-scorebased method developed to predict DTIs using graph embedding, graph mining, and similarity-based techniques. First, DTiGEMS+ integrated multiple drug-drug similarities using similarity network fusion algorithm (SNF) [4], and did the same on multiple target-target after applying a similarity selection procedure. After that, it constructed the final heterogeneous graph by augmenting the known DTIs graph with these two sub-graphs of drug-drug similarity and target-target similarity. Finally, DTiGEMS+calculated the path score features from the full graph and fed them to the ML classifier. 
Current methods that address the DTI prediction problem still suffer from high false-positive rates that need improvement. Thus, in this study, DTi2Vec addresses the DTI link prediction problem in a heterogeneous network using graph embedding and ensemble learning techniques. Figure 2 provides an overview of the pipeline used in our method. The DTi2Vec method's objective is to predict novel DTIs with high accuracy and avoid the limitations associated with literature methods of traditional network analysis. We performed an empirical evaluation by comparing DTI prediction performance of the proposed method DTi2Vec to several network-based state-of-the-art methods using four benchmark datasets and a large-scale FDA_DrugBank dataset. We demonstrated our approach's effectiveness in terms of the AUPR evaluation metric and error reduction rate, and verified novel DTIs predicted by DTi2Vec using reliable databases and scientific literature.

\section{Materials and method DTIs datasets}

We used five datasets for this work, of which four were the "gold standard" Yamanishi_08 [46] datasets. The Yamanishi datasets are families of target proteins (http://web.kuicr.kyoto-u.ac.jp/supp/yoshi/drugtarget/), including (1) G protein-coupled receptors (GPCR), (2) ion channels (IC), (3) nuclear receptors (NR), and (4) enzymes (E). Yamanishi et al. [46] collated the DTI data from several reliable sources, namely KEGG BRITE [47, 48], BRENDA [49], SuperTarget [50], and DrugBank [51] databases released in 2008. They also collated the chemical structures of drugs/compounds from the KEGG LIGAND and KEGG DRUG databases [47, 48]. They then calculated the similarity scores representing drug features for each pair of drugs using [52] by finding the common chemical substructure. They further collected the targets' amino acid sequences from the KEGG GENES database $[47,48]$, then calculated the sequence similarities using the normalized Smith-Waterman scores [53] based on the alignment of protein sequences.

Table 1 Benchmark Yamanishi_08 datasets and FDA_DrugBank dataset statistics

\begin{tabular}{lllllll}
\hline Statistics & \multicolumn{3}{l}{ Benchmark datasets } & \multicolumn{1}{r}{ FDA_DrugBank } \\
\cline { 2 - 5 } & NR & GPCR & IC & Enzyme & \\
\hline Number of drugs & 54 & 223 & 210 & 445 & 1,482 \\
Number of targets & 26 & 95 & 204 & 664 & 1,408 \\
Known DTIs & 90 & 635 & 1476 & 2926 & 9881 \\
Unknown DTIs & 1314 & 20,550 & 41,364 & 292,554 & $2,076,775$ \\
Sparsity ratio & 0.068 & 0.031 & 0.036 & 0.010 & 0.005 \\
\hline
\end{tabular}

The fifth dataset, FDA_DrugBank, used to evaluate DDR [27] and TriModel [37] methods, was retrieved from the 5.0.3 version of the DrugBank database [51]. The FDA_DrugBank dataset only includes the DTIs with Food and Drug Administration (FDA) approval (https:// www.drugbank.ca). We calculated drug similarity SIMCOMP scores, as well as normalized Smith-Waterman scores for the targets.

Table 1 summarizes the statistics of the datasets used in our study. The sparsity ratio is the number of known DTIs divided by the number of unknown DTIs. It reflects that the data is highly imbalanced between negative and positive samples (Table 1).

Link prediction formulation in the heterogeneous network DTI prediction was formulated as a link prediction problem in a heterogeneous graph. The goal is to predict the

Table 2 Fusion functions for learning drug-target edge representations

\begin{tabular}{ll}
\hline Fusion function & Function equation \\
\hline 1. Concatenation & {$[f(d)+f(t)]=f(d) f(t)$} \\
2. Hadamard & {$[f(d) \square f(t)]=f(d) * f(t)$} \\
3. Average & {$[f(d)$ 田 $f(t)]=(f(d)+f(t)) / 2$} \\
4. Weighted-L1 & $\|f(d) \cdot f(t)\|_{1}=|f(d)-f(t)|$ \\
5. Weighted-L2 & $\|f(d) \cdot f(t)\|_{2}=|f(d)-f(t)|^{2}$
\end{tabular}

口 represents element wise matrix multiplication, and $⿴$ represents the average of the two matrices

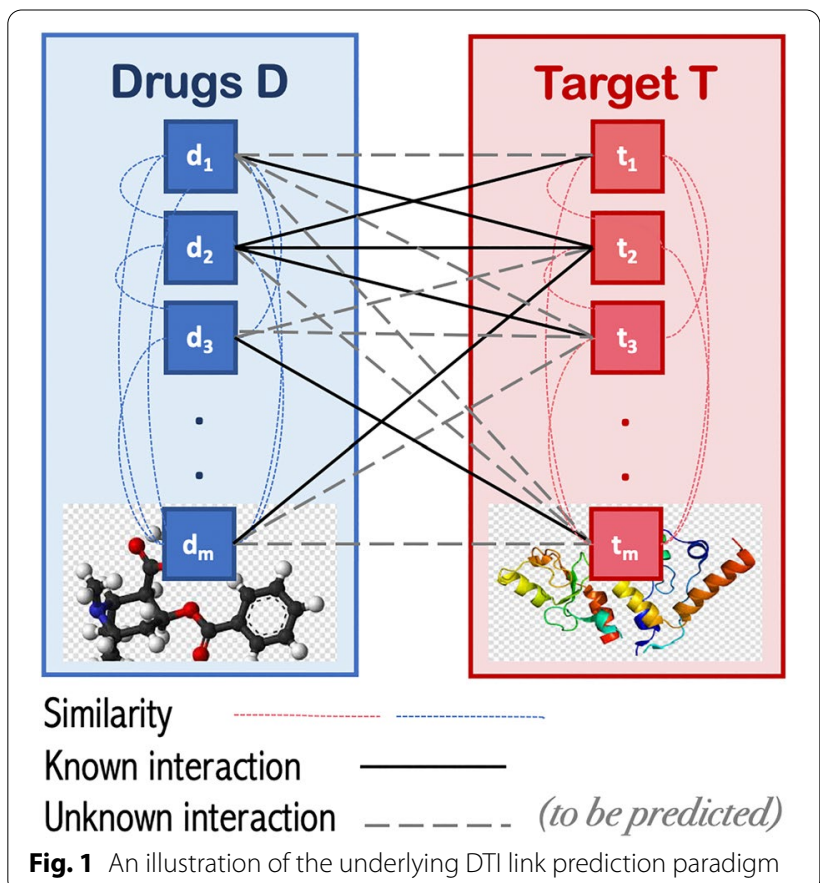




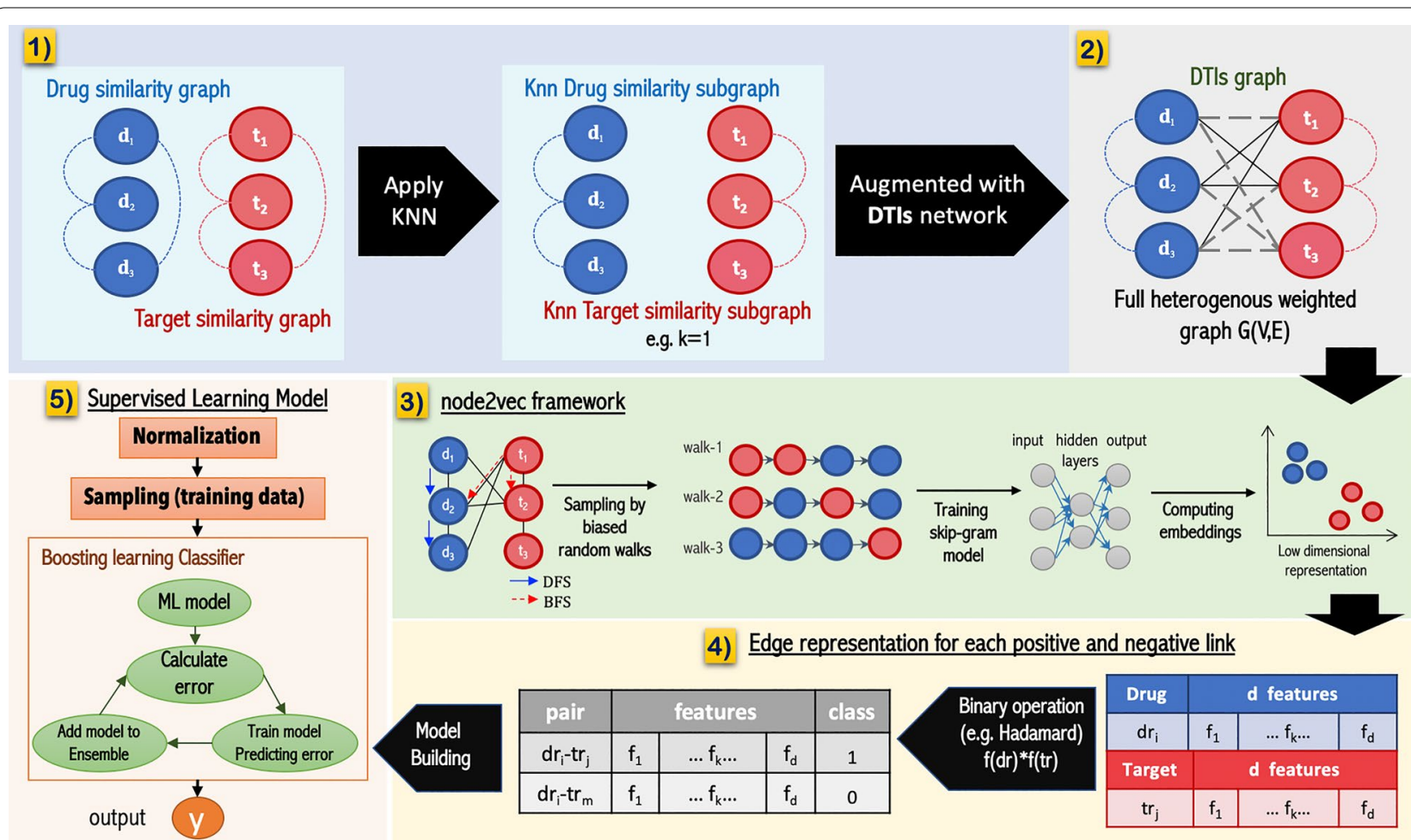

Fig. 2 DTi2Vec Method Flowchart, (1) Filter the TTsim and DDsim graphs, (2) Construct a full DTI network by augmenting the three graphs, (3) Apply the three-step node2vec framework on the full DTI network, (4) Generate edge representation for each drug-target pair, (5) Feed the feature vector (FV) into ensemble boosting classifier to output the class labels

likelihood of a missing link (i.e., edge) between drugtarget nodes in the DTI network, as shown in Fig. 1. We constructed a heterogeneous graph by defining and then augmenting all three networks (the DTIs network, drugdrug similarity network, and target-target similarity network). Thus, we obtained weighted heterogeneous graph $G(V, E)$ that consists of a set of drugs $D=\left\{d_{1}, d_{2}, \ldots, d_{m}\right\}$ and a set of targets $T=\left\{t_{1}, t_{2}, \ldots, t_{n}\right\}$, where $m$ is the number of drugs and $n$ is the number of targets, respectively. Before augmenting each similarity network, we filtered each network separately by applying the $\mathrm{K}$ nearest neighbor (KNN) algorithm to keep the top $\mathrm{k}$ similar nodes as described later in the preprocessing section. In the heterogeneous graph $G$, drug-target edges represent the known DTIs and have weight $=1$, and the drug-drug (or target-target) edges represent the similarity scores and have the weight range of $(0,1]$. We defined the DTIs as an $m * n$ adjacency matrix $Y$ that specify the class labels between each drug-target pair as follow:

$$
y_{i j}=\left\{\begin{array}{l}
1, \text { ifthereisaninteractionbetweend } d_{i} a n d t_{j} \\
0, \text { otherwise }
\end{array}\right.
$$

where $y_{i j}$ corresponds to the $<i, j>$ element of matrix $Y$. The samples with class labels $=0$, called negative samples (i.e., unknown interaction), are constructed by generating an edge between all possible drug-target pairs with no edge connecting them (i.e., unknown interaction). This process reflects a real-life scenario where negative samples are much larger than positive ones. Then, $X$ vector is constructed, where $X=\left\{x_{1}, x_{2}, \ldots, x_{n^{*} m}\right\}$, to represent each data sample (i.e., drug-target pairs feature vector) as will be described later.

\section{Workflow of the DTi2Vec model}

In Fig. 2, we show that implementing DTi2Vec involves five main steps, which are:

1. Preprocessing the drug similarity and target similarity networks.

2. Constructing a heterogeneous network $G(V, E)$ by augmenting the DTI graph (training part) with the k-nearest neighbor drugs subgraph (KNN-DDsim) and the k-nearest neighbor targets subgraph (KNNTTsim).

3. Applying a biased random walk using node2vec framework to generate feature representation (i.e., embedding) for each node (drug and target). 


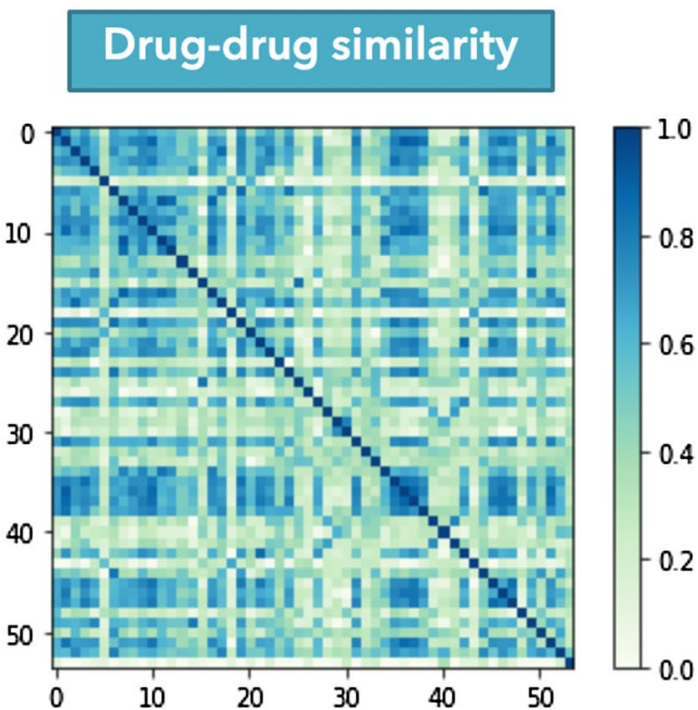

(a)

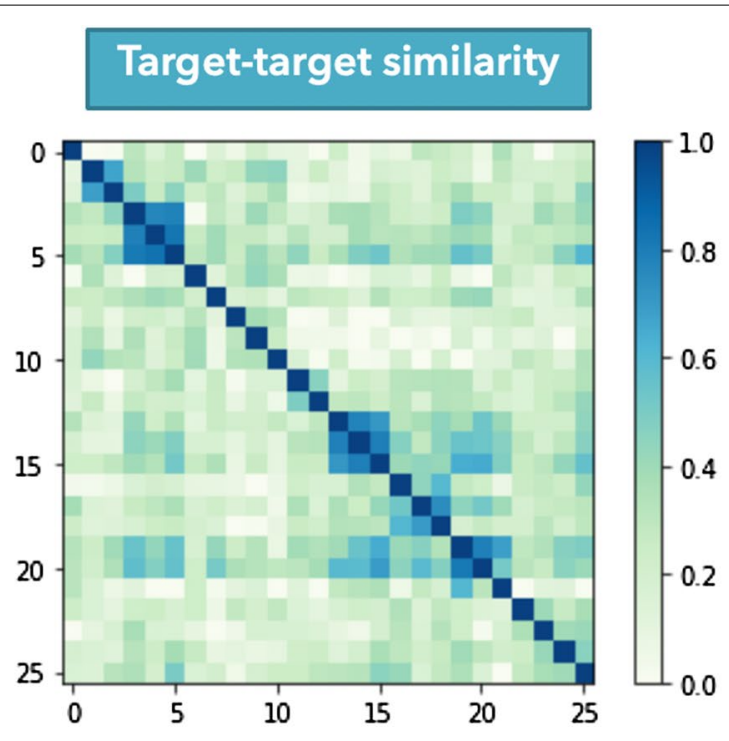

(b)

Fig. 3 Visualization of similarity matrices of the NR dataset, a drug-drug similarity matrix, $\mathbf{b}$ target-target similarity matrix

4. Creating drug-target edge embeddings by generating embeddings for each drug and each target and then combining them using several fusion functions.

5. Classifying the data samples by ensemble learning using boosting classifiers to predict the probability scores for each class.

We provide a detailed explanation of each step below.

\section{Preprocessing}

When the DTI graph includes the similarity scores as edges connecting similar drugs or similar targets, the graph becomes a complex network, especially when dealing with a huge number of drugs or targets. Several similarity scores were very low or shallow, not providing any informative meaning (see the drug-drug similarity and target-target similarity matrices for the NR dataset in Fig. 3). Thus, instead of using all similarity edges for the drug similarity graph, we ranked all similarity scores for each drug in descending order and removed all the drugs except the top-k similar drugs as per the k-nearest neighbors (KNN) algorithm. Finally, we applied the same process to the target similarity network and kept the KNN determined top-k similar targets for each target node. Through this process, we generated a KNN drugs similarity subgraph and a KNN targets similarity subgraph, augmented with the training part of DTIs, as shown in Fig. 2.1. Choosing the $k$ value is not an easy task. If the k setting is too large, it increases the time and memory complexity, making the process computationally expensive. Still, if the $\mathrm{k}$ setting is too small, some useful information could be dropped and affect the performance. Thus, we conducted several experiments to find the optimal number of nearest neighbors $k$, using different values of $\mathrm{k}$. We apply this optimization process by assigning various values to $k$, comparing the model performance under each $k$ value in terms of AUPR in the tenfold $\mathrm{CV}$, and then selecting the $\mathrm{k}$ value based on the best-performing model. Using this technique means we reduced noise or meaningless information, introduced when using all similarity scores, affecting performance. It also decreases the node2vec model's time and memory complexity since the number of the edges for each similarity network is reduced from $m(m-1) / 2$ to $\left(k^{*} m\right)$, where $m$ is the number of the nodes.

\section{Network embedding technique}

Our study applied the graph embedding technique to transform nodes, edges, and features (i.e., similarity) into vector space by converting the graph into a low dimensional space while maximally preserving graph structure, nodes' relationship, and other relevant properties. In the following subsections, we explain in more detail one of the random walk-based embedding techniques, the benefit of using this technique, how it works, and how we generate the feature vector for each drug-target pair.

\section{Random walk-based embedding for features generation}

A random walk is a stochastic process of graph traversing to describe node sequences (i.e., path) consisting of steps 
selected uniformly among the present vertex neighbors. Several graph embedding techniques have been developed based on utilizing random walk in the heterogeneous networks to improve the quality of feature learning (i.e., embedding) $[54,55]$.

In our work, we applied node2vec [56], a semi-supervised feature representation learning technique for nodes in a network that optimizes the objective of preserving the neighborhood. Node2vec is a generalized version of DeepWalk [57] inspired by the famous natural language processing (NLP) technique (word2vec) that treats the sequence of the nodes generated by a short random walk as a sentence and implements Skip-gram [58] to learn features for each node (similar to word). Skip-gram is a language model that consists of a neural network with one hidden layer. This model maximizes the co-occurrence probability among the words appearing within a window in a sentence and then predicts the neighboring words in the sentence.

For this study, we were motivated to apply the node2vec technique instead of DeepWalk because node2vec provides a biased and more flexible random walk strategy that encodes global and local proximities in the sampled path. We achieved this by interpolating two extreme searching strategies: breadth-first search (BFS) and depth-first search (DFS). BFS captures the structural equivalence characteristic while DFS discovers the graph homophily characteristics (i.e., graph communities where nodes are connected close to each other). Two hyperparameters control which search strategy to follow: Return parameter, $p$ and In-out parameter, $q$. Parameter $p$ controls the likelihood of immediately revisiting a node in the walk. In contrast, parameter q controls the search's probability to differentiate between moving inward (i.e., revisiting a node) or outward to nodes that move deeper. [56] provides more detailed information about the node2vec algorithm.

In our method DTi2Vec, after we constructed the full heterogeneous weighted graph $G=(V, E)$ consisting of known DTIs, KNN drug-drug similarity subgraph, and KNN target-target similarity subgraph, we applied the three-step node2vec framework (see Fig. 2.3). The node2vec framework translated each node in the graph $G$ to a low dimensional vector space $R d$, using a mapping function:

$$
f: V \rightarrow R_{d}, \text { where } d<|v|
$$

while preserving the graph structure and some sort of node similarity based on the network topology, resulting in obtaining a FV representation (i.e., latent feature vectors) for each drug and target nodes. Moreover, since more properties encoded by the embedder leads to better results in the downstream task performance, we utilized several node2vec hyperparameters to improve the feature representations' quality. Thus, we performed a grid search on multiple parameters to identify those values that provide the best performance using the training data during the tenfold $\mathrm{CV}$ process for each dataset separately. We created a dictionary of all the different hyperparameter values for the grid search, which feeds all combinations through the model for testing. We applied GridSearchCV by first initializing the node2vec hyperparameters' values from the dictionary, then using tenfold $\mathrm{CV}$ to obtain the model performance using the training data. Then, a second-round is started by changing the hyperparameters' values from the dictionary and applying the same steps. Once all the values are tested, the grid search cycle finishes. The hyperparameter combinations that give the best performance during the training stage are selected. We applied this GridSearchCV procedure on each dataset separately except for the DrugBank dataset, where we used RandomizedSearchCV to tune the hyperparameters. Running these experiments on the huge DrugBank dataset will require excessive run time and large memory if GridSearchCV is applied. Therefore, we applied these two algorithms to identify the optimal values for the different datasets' hyperparameters. This allowed us to identify the optimal values of these parameters, including embedding dimension d (i.e., the feature representation length); walk-length, the number of walks per source node; the number of the walk num-walk; and the worker number that scales and parallelizes the walks from each source node, which speeds up the feature generation process. Additional file 1: Table S1 provides the tested parameter values and indicates the optimal parameter values for each dataset.

\section{Edge representation learning}

After the node2vec framework generated an embedding matrix $\mathrm{f}$ of size $|V| x d$ (Vis the number of vertices and $d$ is the feature dimension), we extracted the embedding (i.e., FV representation) for each drug and each target in the DTI network. Then, because our goal is to predict drugs and targets potentially linked, we created an embedding for each drug-target pair (i.e., edge). We accomplished this task by applying a fusion function between two FVs, because the fusion function combines two FVs to obtain a single FV. So, given two nodes drug $\left(d_{i}\right)$ and target $\left(t_{j}\right)$ in $G(V, E)$, we generated edge feature representation $g\left(d_{i}, t_{j}\right)$ by applying several fusion functions over the corresponding nodes FV representation $f\left(d_{i}\right)$ and $f\left(t_{j}\right)$ such that $g: V x$ $V \rightarrow>R^{d}$, where $d$ is the representation size for the pair $(\mathrm{d}, \mathrm{t})$ (Table 2). We applied these fusion functions to any possible drug-target pair, including the unknown DTIs (i.e., when the edge does not exist between this drug and target). 
Note, in the fusion function step (Fig. 2, panel 4) there is no dimensionality reduction. In all fusion function cases the FV sizes remained the same (where $d^{\prime}=d$ ), except in the concatenate fusion function case, where the FV sizes increased $\left(d^{\prime}=2 d\right)$ since it connects the drugs' FV and targets' FV for each drug-target edge (i.e., by joining the feature representation $f\left(d_{i}\right)$ and $f\left(t_{j}\right)$ in one series). We specified the node FV dimension (between 32 and 128) that has been used for each dataset in Additional file 1: Table S1. Therefore, the final FV size for each edge was between 32 and 256 (note, $128 \times 2=256$, in the concatenate fusion function case).

\section{ML predictive model}

After extracting all the drug-target pairs' features, we prepared the FVs to be fed into the ML classification model by first normalizing the training and test datasets separately using min-max normalization [59] and then oversampling the training data (explained below). We used min-max normalization [59] to ensure that all features are equally treated by the classifier where all features get transformed into a given range, which is $[0,1]$ in our case. We applied min-max normalization as follows: first, we define the min-max normalization scalar, fit this scalar on the training data that saves the normalization parameters, and then transform the training data features. The last step is to normalize the features of the test data using the training normalization parameters by applying the scalar that was fitted.

\section{Sampling techniques for imbalanced data}

Table 1 shows that the number of unknown DTIs is much larger than the known DTIs in all datasets. This issue needs to be dealt with as ML classifiers face a problem when predicting based on imbalanced data, i.e., the $\mathrm{ML}$ models classify most test samples into the majority class when the minority class lacks information. We solved this problem by applying random oversampling [60] on the minority class (i.e., positive known DTIs) to obtain the same number of DTIs as the majority class (negative unknown DTIs) in the training data. This technique's implementation is done using an imblearn python package [61].

\section{Boosting learning model}

Boosting converts a family of weak learners into strong learners by combining weak learners to build ensembles: a more efficient learning algorithm that achieves better prediction performance. In boosting algorithms, the most common type of weak learner used is decision trees. Each decision tree decides what features are essential for the next model and tries to correct any mistake introduced by the previous model in a sequential process.
Figure 2.5 provides an overview of how the boosting classifier works.

In DTi2Vec, supervised ML models are utilized based on two ensemble classifiers, Adaptive Boosting (AdaBoost) and eXtreme Gradient Boosting (XGBoost) classifiers [62], that are implemented respectively for DTIs prediction. AdaBoost is implemented using python library scikit-learn [63], and the XGBoost classifier is implemented using an optimized distributed gradient boosting library, called XGBoost [64]. AdaBoost uses decision trees as weak learners. XGBoost uses regression trees or CART (Classification and Regression Trees), which use continuous scores assigned to each leaf and then sum them up and provide the final prediction instead of having equal weight as in the decision trees.

XGBoost provides parallel tree boosting, which enhances performance in terms of speed. For each classifier, we performed hyperparameter optimization using tenfold CV on the training data, then tested the models using the test set to determine the set of optimal hyperparameters. We selected the model with the best performance in terms of AUPR and the corresponding set of hyperparameters. However, XGBoost tuned more hyperparameters than AdaBoost, such as the weighted regularization parameters (e.g., lambda and alpha), the tree construction algorithms, subsample ratio, etc. The most critical parameters for both classifiers include the number and maximum depth of trees, the learning rate, and the number of features and the function needed to attain a quality split.

The feature embeddings that are learned for each node and then generated for each edge (i.e., drug-target pair) are the input that is fed into these two boosting classifiers to predict the likelihood of their interactions. We constructed the ML prediction model by providing this FV $X$ for both positive and negative data with their labels $Y$, as either known DTIs to represent positive labels or unknown DTIs that are treated as negative labels. This procedure is done for each dataset separately.

\section{Results and discussion}

This section describes the evaluation protocols, the conducted experiments, and the results of our DTI prediction experiments using FDA_DrugBank and four benchmark datasets. We further compared the performance of our model, DTi2Vec, with select stateof-the-art methods. We also validated several novel DTIs predictions using scientific literature and 'reliable' databases.

\section{Evaluation metrics and protocols}

Several performance metrics are used in the literature to compare the performance of binary classification 
methods. However, since the datasets we used are highly imbalanced, typical accuracy measures are not accurate. Thus, the area under the receiver operating characteristic (ROC) curve (AUC) [65] and the area under the precision-recall curve (AUPR) [65] are standard evaluation metrics used in DTIs prediction even though the AUC is over-optimistic when dealing with such a problem. Moreover, AUPR is considered more informative and provides better assessment, in such cases, by separating the predicted scores of known interactions from predicted scores of the unknown interaction. Thus, in our study, we mainly focus on the AUPR metric for performance evaluation. AUC and AUPR are defined and computed on the testing data. To obtain AUC and AUPR, we calculated the true positive rate (TPR) (also referred to as recall or sensitivity) and the precision [66], as shown in Eqs. 3 and 4, respectively.

$$
\begin{aligned}
& \text { Recall }=T P R=T P /(T P+F N) \\
& \text { Precision }=T P /(T P+F P)
\end{aligned}
$$

The precision is the ability of the classifier not to label as positive a sample that is negative (i.e., the ratio of true positive (TP) over the total of TP and false positive (FP)). The recall is the classifier's ability to find all the positive samples (i.e., the ratio of TP over the total of TP and false negative (FN)). We constructed the ROC curve based on calculations using different recall and false predictive rate (FPR) values of different thresholds, then calculated the area under the ROC curve. Similarly, we constructed the AUPR curve based on calculations using different precision and recall values at different cut-offs, then calculated the area under this curve. The closer the value of AUC and AUPR is to 1, the better performance. We also calculated the error rate (ER) and the relative error rate reduction for the best performing model compared to the second-best performing model $(\Delta \mathrm{ER})$, as defined in Eqs. 5 and 6, respectively:

$$
\begin{aligned}
& E R=1-A U P R \\
& \triangle E R=\left(E R_{2}-E R_{1}\right) / E R_{2}
\end{aligned}
$$

where $E R_{1}$ and $E R_{2}$ are the error rate of the best and second-best methods, respectively. We evaluated the prediction performance of our method by applying a random setting. The random setting ensures that all the drugs and the targets are seen in the training data, which means every target and drug has at least one interaction in the training data. To facilitate the performance comparison on the benchmark Yamanishi_08 and FDA_DrugBank datasets with state-of-the-art methods, we followed these methods' evaluation setup by conducting stratified tenfold CV for each dataset separately. We applied random CV setting where random pairs of (drug-target) are removed to be in the test data. Therefore, we randomly split the data into ten subsets in a stratified fashion wherein each subset must include the same percentage of negative and positive samples (i.e., enforcing the positive and negative class distributions in each fold to match the distribution in the whole data). We used this stratified strategy since the data is imbalanced because using the standard CV may result in some subsets without a positive label, which affects the performance and causes a computational error. We used nine subsets (with positive and negative samples) in each training stage to fit the model and kept the remaining subset, the testing data, to evaluate the model. We repeated this process ten times using each subset in the testing part. All results are reported based on the overall performance by averaging the model performance in the 10 test subsets. It is worth mentioning that we removed all the edges of the known DTIs, from the constructed graph G, in the test data before applying the node2vec technique.

Although the random setting, tenfold CV, is the most popular and widely used in computational methods, it will mimic the real application scenario when there are no known interactions for the new drugs. Therefore, we apply a new drug setting (i.e., leave drugs out) by splitting the data to have only part of the drugs available in the training phase, while the other known DTIs in testing contain drugs that have no known DTIs in the training data. We repeated this process ten times to simulate a tenfold $\mathrm{CV}$ but by holding new drugs in each round for testing and then averaging the results. We evaluated DTi2Vec using the new drug setting, but with the same node2vec hyperparameters and XGBoost classifier hyperparameters (the only classifier used for the "new drug" experiments) used for the random setting experiments. We also tested different node2vec embedding dimensions and chose the dimension with the best results.

We executed all experiments on a Linux Ubuntu machine with 112 processors, running some parts of our implementation in parallel, such as generating embedding using node2vec or in XGBoost classifier. We used python 3.7 for the implementation, importing some important libraries mentioned above in previous sections.

\section{DTi2Vec experiments performance}

Table 3 provides the results we obtained for our experiments. For each dataset, separately, DTi2Vec was evaluated in terms of AUPR using two ensemble learning classifiers for different sets of FVs learned by applying 
Table 3 Performance of DTi2Vec in terms of AUPR using AdaBoost and XGBoost classifiers on each dataset with multiple FVs generated by applying different edge representation functions for the random CV setting, and averageAUPR for each fusion function across all datasets for each classifier

\begin{tabular}{|c|c|c|c|c|c|c|c|}
\hline \multirow[t]{2}{*}{ Model } & \multirow{2}{*}{$\begin{array}{l}\text { Fusion function } \\
\text { (FF) }\end{array}$} & \multicolumn{4}{|c|}{ Yamanishi_08 datasets } & \multirow[t]{2}{*}{ FDA_DrugBank } & \multirow[t]{2}{*}{ AVG AUPR per FF } \\
\hline & & NR & GPCR & IC & $E$ & & \\
\hline \multirow{5}{*}{$\begin{array}{l}\text { DTi2Vec } \\
\text { AdaBoost }\end{array}$} & Concatenate & $0.74(0.145)$ & $0.83(0.039)$ & $0.97(0.010)$ & $0.97(0.01)$ & $0.77(0.014)$ & 0.856 \\
\hline & Hadamard & $0.85(0.127)$ & $0.89(0.037)$ & $0.93(0.020)$ & $0.96(0.007)$ & $0.82(0.009)$ & 0.89 \\
\hline & Average & $0.64(0.132)$ & $0.78(0.054)$ & $0.90(0.029)$ & $0.92(0.007)$ & $0.75(0.022)$ & 0.798 \\
\hline & Weighted L1 & $\underline{\mathbf{0 . 9 2}}(0.082)$ & $0.84(0.052)$ & $0.93(0.017)$ & $0.96(0.008)$ & $0.82(0.009)$ & 0.894 \\
\hline & Weighted L2 & $\underline{0.92}(0.082)$ & $0.84(0.054)$ & $0.94(0.018)$ & $0.96(0.008)$ & $0.82(0.008)$ & $\underline{0.896}$ \\
\hline \multirow{5}{*}{$\begin{array}{l}\text { DTi2Vec } \\
\text { XGBoost }\end{array}$} & Concatenate & $0.72(0.118)$ & $0.87(0.039)$ & $\underline{\mathbf{0 . 9 8}}(0.0096)$ & $\underline{\mathbf{0 . 9 8}}(0.007)$ & $0.82(0.011)$ & 0.87 \\
\hline & Hadamard & $0.81(0.115)$ & $\underline{\mathbf{0 . 9 0}}(0.036)$ & $0.93(0.017)$ & $0.97(0.006)$ & $\underline{\mathbf{0 . 8 8}}(0.0087)$ & 0.898 \\
\hline & Average & $0.68(0.142)$ & $0.81(0.042)$ & $0.91(0.024)$ & $0.95(0.009)$ & $0.78(0.020)$ & 0.826 \\
\hline & Weighted L1 & $0.88(0.107)$ & $0.84(0.055)$ & $0.94(0.014)$ & $0.97(0.006)$ & $0.87(0.009)$ & 0.9 \\
\hline & Weighted L2 & $0.89(0.103)$ & $0.84(0.055)$ & $0.94(0.014)$ & $0.97(0.006)$ & $0.87(0.009)$ & 0.902 \\
\hline
\end{tabular}

AUPR in bold font with underline indicate the best result in each dataset, and the italic values between parentheses are the standard deviations

five edge fusion functions. We calculated the standard deviations (std) to evaluate each models' robustness. This process allowed us to test our methods' performance in multiple experiments before selecting the model with the best-obtained results. All std values reflected robustness in the performance of DTi2Vec (i.e., std $<0.05$ ) except when using the very small NR dataset.

The results are not entirely consistent among the classifiers and FVs across all datasets (specifically NR dataset due to its excessive small size) (see Table 3). However, it shows the XGBoost classifier achieved the best performances except when using the NR dataset, with which the AdaBoost classifier obtained the best performance. The reason we obtained this result may be multifold. First, compared to the XGBoost classifier, Adaboost performs worse when including irrelevant features, and the noise is high, which may be the likely scenario when dealing with larger datasets. Thus, AdaBoost performed better in the smallest dataset NR. XGBoost is more robust since it has a regularization parameter that successfully reduces variance. Furthermore, the main advantage of XGBoost is its speed due to the implementation of parallel processing, making it significantly faster than the AdaBoost classifier.

Beyond the differences observed when using AdaBoost and XGBoost, we further observed that DTi2Vec's overall performances are drastically higher for the datasets grouped based on the target proteins family (i.e., Yamanishi_08 datasets) than for the FDA_DrugBank dataset. Also, with all FVs, both classifiers delivered better performance when dealing with the larger datasets, IC and E, than NR and GPCR (for Yamanishi_08 datasets), suggesting applying node2vec to large graphs generate high quality and more meaningful embedding.
The best results obtained for:

1. The NR dataset was from FVs generated using the WL1 and WL2 functions using AdaBoost,

2. The GPCR dataset was generated from FVs using the Hadamard function using XGBoost, and

3. IC and Enzyme datasets were generated using FVs from the Concatenate functions using XGBoost.

Thus, we did not observe any explicit link between the functions used to generate FVs and the individual performances observed with each dataset. However, the Average function obtained the worst performance for each dataset using both classifiers. Therefore, for any further analysis or optimization, we will exclude the average function. Also, using FVs from the Concatenate functions seemed to work better with the larger datasets (IC and Enzyme). Thus, for the FDA_DrugBank dataset, we expected to obtain the best result using the Concatenate function; however, we got better results overall for this more complex dataset using Hadamard with XGBoost and the second-best using the WL1 and WL2 functions with the same classifier. Using the AdaBoost classifier achieved lower performances than when using XGBoost, but the Hadamard, WL1, and WL2 functions performed the same and achieved better results overall. The results suggest Hadamard, WL1, and WL2 are the more stable functions that produce good quality FVs with the DrugBank dataset. For further analysis and to gain more insights into the impact of fusion function choices, we calculated the average performance in terms of AUPR for each fusion function across all datasets for each classifier separately. 


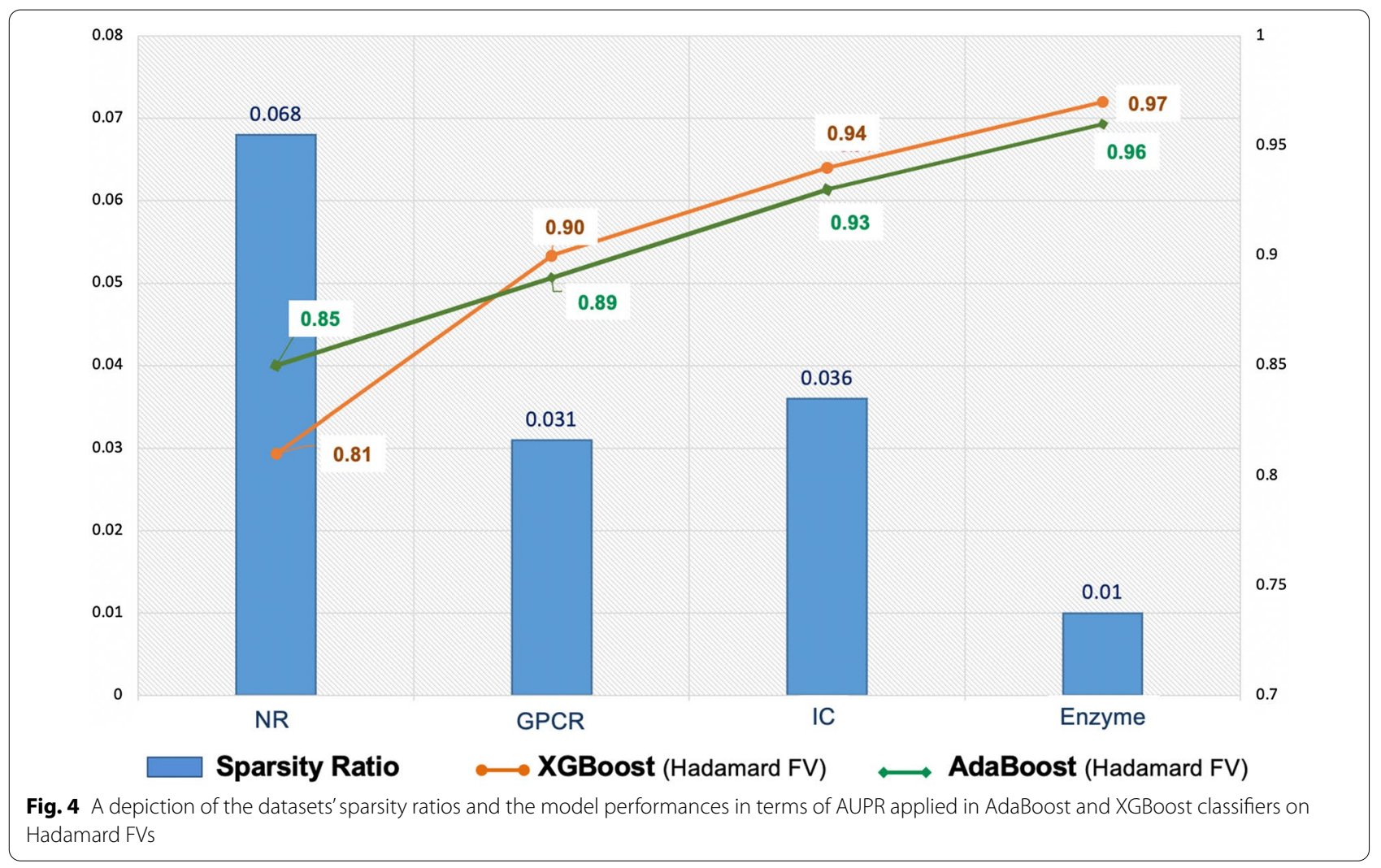

With this average, we have a clear vision about the best function performance. The average performances have the same order across all fusion functions for both classifiers, as reflected in the last column in Table 3. Overall, Weighted-L2 gives the best performance based on average AUPR across all datasets for both classifiers. Weighted-L1 (second-best) and Hadamard (third-best) obtained average results very close to the best performing function WL2. The overall results indicate that WL2 and WL1 are the more highly stable functions and then the Hadamard function.

Furthermore, based on the sparsity ratio for each dataset (see Table 1) and the results of the Yamanishi_08 datasets in Table 3, we can see the link between the sparsity issue and the model performances. We observe that the model performance is low when the sparsity ratio is high, as is the case for the NR dataset. On the contrary, when the sparsity ratio decreases, the model performance increases, as seen in GPCR and Enzyme datasets. We can see that although the model has better performance using IC, the sparsity ratio is larger than for the GPCR dataset. The number of known interactions in the IC dataset being much larger than in GPCR, suggests that the sparsity ratio and the number of known interactions may affect performance. Nonetheless, the overall performance is good even though the sparsity ratio is high since DTi2Vec can effectively deal with sparsity problems and imbalance issues. Figure 4 illustrates the connection between the sparsity ratio and the performances in terms of AUPR applied in two different classifiers.

\section{Comparing the performances of DTi2Vec and the state-of-the-art methods}

Here we compare DTi2Vec's performance on the NR, GPCR, IC, E, and FDA_DrugBank datasets with five state-of-the-art methods, which include DTiGEMS + [28], TriModel [37], DDR [27], DNLMF [43], and NRLMF [45]. We selected these methods to give a comprehensive comparison and a broad perspective of our method prediction performance against the most representative and successful network-based DTIs prediction methods. Since DTiGEMS $+[28]$ is more recent and is the extension method of DTiGEM [29] that achieved better results, we exclude the latter from the comparison.

Table 4 shows DTi2Vec outperforms all the stateof-the-art methods based on it obtaining the highest averageAUPR for the four Yemanashi_08 datasets. It also obtained the highest averageAUPR for all datasets, including the FDA_DrugBank dataset, which is 3\% higher than the second-best method (DTiGEMS+) in the first case and $2 \%$ higher than the third-best method 
Table 4 Prediction performances for DTi2Vec and all comparison methods across all benchmark datasets

\begin{tabular}{|c|c|c|c|c|c|c|c|}
\hline \multirow[t]{2}{*}{ Dataset } & \multirow[t]{2}{*}{ Metric } & \multicolumn{6}{|l|}{ Method } \\
\hline & & NRLMF & DNILMF & DDR & TriModel & DTiGEMS+ & DTi2Vec \\
\hline \multirow[t]{2}{*}{ Yamanishi_08 datasets } & AvgAUPR & 0.80 & 0.78 & 0.87 & 0.88 & $\underline{0.92}$ & $\underline{0.95}$ \\
\hline & AvgAuC & 0.95 & 0.95 & 0.96 & 0.98 & $\underline{0.99}$ & $\underline{1.00}$ \\
\hline \multirow{2}{*}{$\begin{array}{l}\text { All datasets } \\
\text { (Yamanishi_08 and FDA_DrugBank) }\end{array}$} & AvgAUPR & 0.72 & 0.69 & 0.82 & 0.84 & $\underline{0.86}$ & $\underline{0.93}$ \\
\hline & AvgAuC & 0.94 & 0.95 & 0.96 & 0.98 & $\underline{0.99}$ & $\underline{0.99}$ \\
\hline \multirow{2}{*}{$\begin{array}{l}\text { FDA_DrugBank } \\
\text { (Hold-out test set) }\end{array}$} & AUPR & 0.34 & 0.31 & 0.63 & $\underline{0.66}$ & 0.62 & $\underline{0.82}$ \\
\hline & $A \cup C$ & 0.93 & 0.95 & 0.97 & 0.99 & 0.97 & 0.99 \\
\hline
\end{tabular}

We rounded off all results to two decimal places. The bold underlined font indicates the best result in each category, while underlined values indicate the second-best outcome

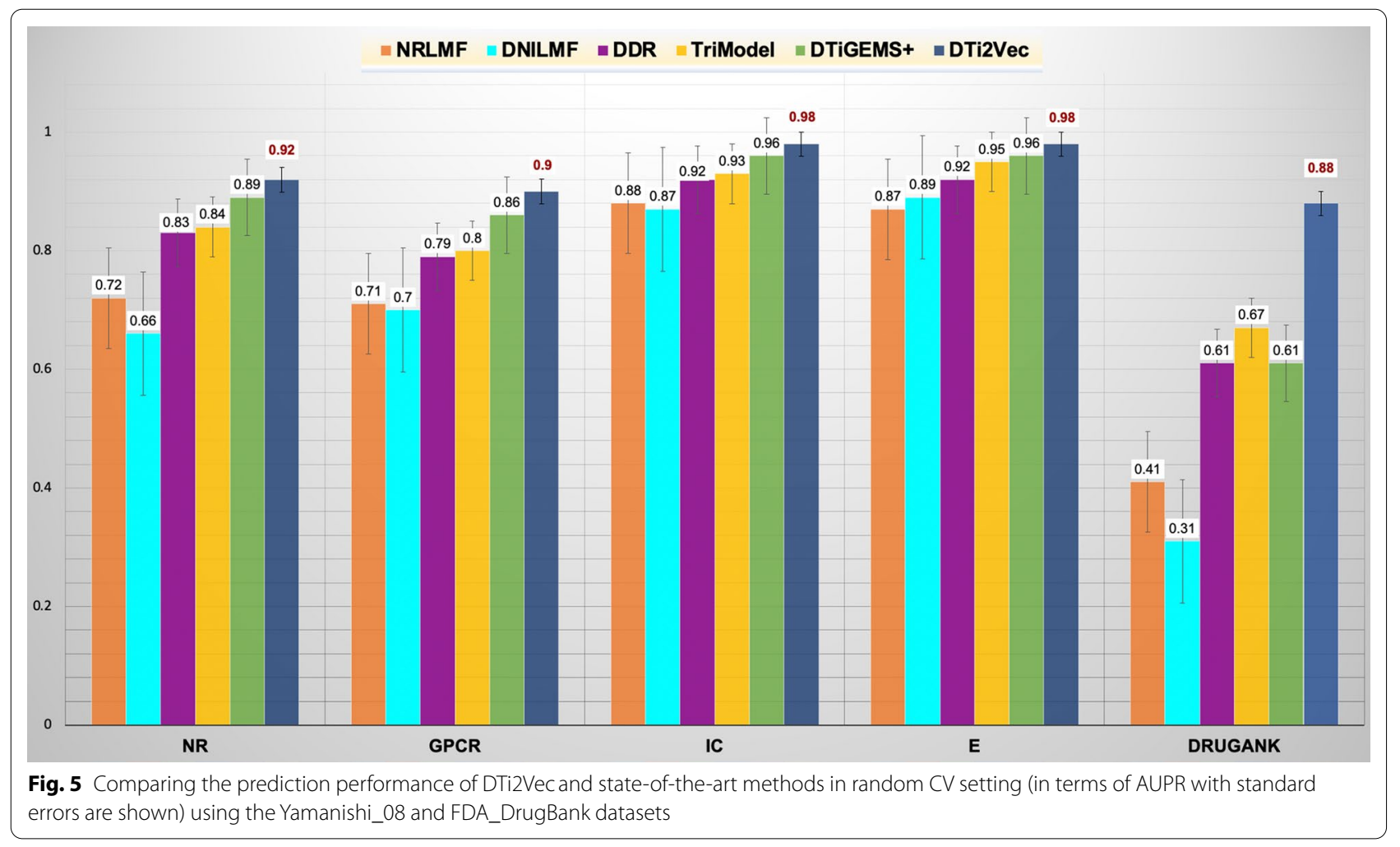

(DTiGEMS+) in the second case. It also achieved the best averageAUC in the two cases. However, since the AUC results are already high in most other methods, DTi2Vec only showed a slight improvement in this metric (see Additional file 1: Table S2), but this is not the case for the AUPR performance metric (see Table 4). We separated the calculation of averageAUPR to have two averages, one across Yamanashi_08 datasets and the other across all datasets, which includes the FDA_DrugBank dataset, to show our method's high performance in both cases. We applied this evaluation because most of the current methods have not achieved high performance using FDA_DrugBank in terms of AUPR, and we show our method performs better with and even without the FDA_DrugBank dataset. Table 4 shows the best and second-best results in each category.

As shown in Fig. 5, DTi2Vec outperformed the other state-of-the-art methods in terms of AUPR 0.92, 0.90, 0.98, 0.98, 0.88 for the NR, GPCR, IC, Enzyme, and FDA_DrugBank datasets, respectively. Additional file 1: Table S3 provides each fold result for 10 -folds CV with the standard deviation of AUPR in 10-folds CV for each dataset (to show the stability of the results). For the NR, GPCR, IC, and Enzyme datasets, DTi2Vec outperformed the second-best method (DTiGEMS+) by $3 \%$, $4 \%, 2 \%$, and $1 \%$, respectively. For the FDA_DrugBank 
dataset, DTi2Vec outperformed the second-best TriModel by $21 \%$. Furthermore, to show that our results are statistically significant compared to the other methods, we applied the Wilcoxon test $[67,68]$ (a nonparametric statistical test that compares two paired groups). The Wilcoxon test comes in two versions, the Signed Rank test or the Rank Sum test, based on the individual AUPR values for each fold in the tenfold CV (see Additional file 1: Table S3). We demonstrate that the DTi2Vec results reflect an increase in the performance that is statistically significant compared to the secondbest method (DTiGEMS +, TriModel) with probability values (p-values) $<0.05$ obtained over GPCR, IC, E, and FDA_DrugBank datasets as 0.021, 0.014, 0.001 and 0.0002 , respectively, except for the NR dataset which has $\mathrm{p}$-value $>0.05$.

We calculated the AUPR score error rate (ER) and the relative reduction of the AUPR score error rate of the best method (DTi2Vec) relative to the second-best method, defined previously in Eqs. 4 and 5, respectively, to show DTi2Vec's performance improvement and its robustness. Using obtained AUPR of tenfold CV experiments, the $\mathrm{ER}=8 \%, 10 \%, 2 \%, 2 \%$, and $12 \%$ for NR, GPCR, IC, E, and FDA_DrugBank, respectively. For predicting DTIs in NR, GPCR, IC, and Enzyme, our method DTi2Vec significantly reduces AUPR error relative to the next best method (DTiGEMS+) by 33\%, 29\%, 50\%, and 33\%, respectively. For the FDA_DrugBank dataset, the relative reduction of the AUPR error obtained by DTi2Vec relative to the next best method (TriModel) is 63\%. Our method, DTi2Vec, consistently reduced the relative error rate compared to the state-of-the-art methods.

Nonetheless, we performed one more experiment using hold-out test data to demonstrate DTi2Vec's prediction reliability. We used the FDA_DrugBank dataset and split the data into three sets for training, validation, and testing. First, we initialized the hyperparameter values using the values obtained in the optimization process for the first experiment. Then we generated node2vec embeddings and used these embeddings to train the model. Next, we evaluated DTi2Vec on the validation set. We repeated this process several times to test random hyperparameter values for tuning, similar to Randomized Search. Finally, we generated embeddings using the optimized hyperparameter set and evaluated the model prediction performance on the test set. Using the random prediction setting, DTi2Vec achieves the highest AUPR on the hold-out test data compared to state-of-the-art methods (Table 4). Relative to the second-best method (TriModel), DTi2Vec reduces the AUPR error rate for the FDA_DrugBank dataset by $47 \%$.

For the other experimental setting, when DTi2Vec predicted interactions for the new drugs, we obtained the results using all sets of FVs generated using the fusion functions except for the AVG function that always performed the worst, and we provided these results in the Additional file 1: Table S4. In addition, we compared the results with the same state-of-the-art methods but excluding DTiGEMS+ since it does not handle new drug settings. The best-obtained results when predicting new drugs are reflected in Fig. 6, which shows DTi2Vec outperformed all the state-of-the-art methods based on average AUPR across all datasets. Moreover, the significantly higher performance compared to the secondbest (TriModel) is associated with the larger datasets, IC (AUPR 12\%), Enzyme (AUPR 15\%), and DrugBank (AUPR 26\%). The new drug setting results for all the state-of-the-art methods are taken from [37].

The last evaluation process we performed to show the practicality of the predictive power of DTi2Vecin real scenarios by establishing the ability of our model to reposition a specific drug other than a hub drug node. This ability is important as hub nodes will likely not be the subject of drug research and development as they are usually well-studied. We apply this assessment: first, we calculate the average precision for DTI prediction at each drug. Second, we average this value (i.e., the average precision) over 10 -folds. Finally, we calculate the mean average precision (MAP) [69] as the mean of 10 -folds average precision for each drug across all drug nodes in the graph. We show that DTi2Vec achieves high MAP values, over NR, GPCR, IC, E, and FDA_DrugBank datasets as $0.96,0.82,0.91,0.91$, and 0.72 respectively. We conclude that the hub nodes do not likely drive our model's overall performance from these results.

In summary and based on all reported results, DTi2Vec outperformed all state-of-the-art methods in two independent datasets: Yamanashi_08 datasets and the FDA_ DrugBank dataset and proved its general applicability, and declared its effectiveness in the prediction of DTIs.

\section{Novel DTI prediction and validation}

To further demonstrate and analyze the capability of DTi2Vec, we performed a complimentary evaluation of its ability to predict new DTIs for each dataset and then validate the DTIs. To predict the new DTIs, we used all positive samples to train the model and divided the negative samples into training and test sets. The predicted DTIs (unknown in the original data but predicted to be positive) with high scores in the testing data are ranked based on their scores. We report the top 5 ranked DTI for each dataset separately. We manually confirmed those novel candidate DTIs using scientific literature and biomedical databases, including DrugBank [70], KEGG [47], ChEMBL [71], PubChem [72], Comparative Toxicogenomics Database (CTD) [73], MATADOR 


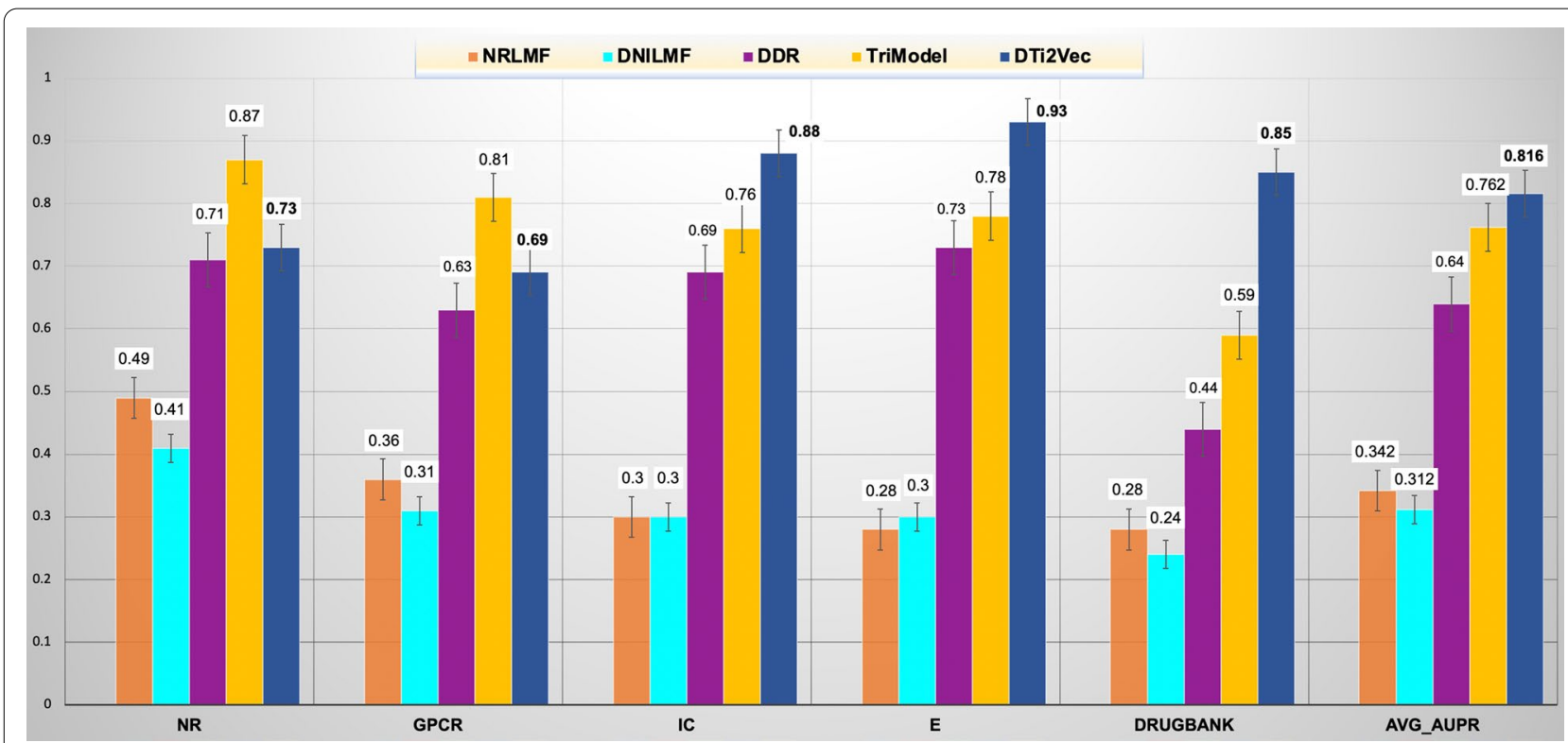

Fig. 6 Comparing the prediction performance of DTi2Vec and other state-of-the-art methods when using new drug settings (in terms of AUPR using the Yamanishi_08 and FDA_DrugBank datasets, and the average AUPR across all datasets)

and SuperTarget [50], and Uniprot knowledgebase [74]. Table 5 shows the top 5 ranked novel DTIs for Yamanishi_08 and FDA_DrugBank datasets with the validation evidence. When we did not find any evidence, we marked the evidence as unknown since there is no confirmation that this interaction exists. We confirmed 21 out of 25 $(84 \%)$ of the newly predicted DTIs as known interaction (i.e., this includes the top 5 ranked DTI for each of the five datasets used). Here, it is important to note that the DTIs data was last updated in 2008; this may be why we managed to confirm so many of the novel DTIs.

\section{Key strength of DTi2Vec}

This section highlights some reasons for improvements in DTi2Vec performance compared to the current stateof-the-art methods and how our results show high promise in identifying novel DTIs.

\section{The advantages of DTi2Vec over DTIGEMS+}

DTi2Vec is a simple yet effective method that achieves less error-prone results using one type of drug similarity and target/protein similarity augmented to DTIs. In contrast to other graph-based methods such as DDR and DTiGEMS+, it leverages from the full weighted heterogeneous graph $\mathrm{G}$, without the complexity of selecting and integrating multiple kernels (i.e., similarities) that require much effort and time. However, it can utilize different types of similarity that can be augmented to the full graph $\mathrm{G}$ and then follow the same steps.
DTi2Vec has several advantages over our previous method (DTiGEMS+, the second-best method) using the Yamanishi_08 datasets, even though they have some similarities in the feature extraction pipeline, including applying the node2vec technique. DTi2Vec has an advantage over DTiGEMS + in five major points:

1. DTi2Vec performed much better in the most extensive dataset DrugBank, by increasing the prediction performance by $27 \%$ compared to DTiGEMS+ in terms of AUPR.

2. DTi2Vec has a more straightforward pipeline with fewer steps but is more effective, faster, and require less memory than DTiGEMS + mainly because:

a) DTi2Vec constructs just one graph to auto-generate features for each drug-target edge, while DTiGEMS + constructed three graphs for the handcrafted feature extraction for each drug-target pair.

b) DTi2Vec utilized single drug-drug similarity and target-target similarity while DTiGEMS + calculated and utilized multiple drug-drug and target-target similarities then applied similarity selection and integration. All these steps increase the time and memory complexities.

3. Both DTi2Vec and DTiGEMS + applied node2vec on the whole heterogeneous DTIs network, but they used the embeddings for each node in different ways. The DTi2Vec method takes advantage of the automatically learned feature representations in low 
Table 5 The highly ranked (top-5) candidate novel DTIs obtained by DTi2Vec for each dataset verified with evidence from databases and published literature

\begin{tabular}{|c|c|c|c|c|c|}
\hline$\#$ & DrugID & Drug name & TargetID & Target name & Validation evidence \\
\hline \multicolumn{6}{|c|}{ NR dataset (KEGG IDs) } \\
\hline 1 & D00690 & Mometasone furoate & hsa2908 & NR3C1 (Glucocorticoid receptor) & PMID:8439518 \\
\hline 2 & D00075 & Testosterone & hsa5241 & PGR (Progesterone Receptor) & $\begin{array}{l}\text { PMID: } 23229004 \\
\text { PMID: } 23933754 \\
\text { C: } 386630\end{array}$ \\
\hline 3 & D00554 & Ethinyl estradiol & hsa2100 & ESR2 (Estrogen Receptor 2) & CTD: D004997 \\
\hline 4 & D00327 & Fluoxymesterone & hsa5241 & PGR (Progesterone Receptor) & Unknown \\
\hline 5 & D00348 & Isotretinoin & hsa5915 & RARB (Retinoic acid receptor beta) & KG: D00348- DG01604, C \\
\hline \multicolumn{6}{|c|}{ GPCR dataset (KEGG IDs) } \\
\hline 1 & D05792 & Salmeterol & hsa153 & ADRB1 (adrenoceptor beta 1) & Unknown \\
\hline 2 & D04625 & Isoetharine & hsa154 & ADRB2 (Adrenoceptor Alpha 1B) & PMID:21948594 \\
\hline 3 & D02358 & Metoprolol & hsa154 & ADRB2 (Adrenoceptor Alpha 1B) & DB:DB00264 \\
\hline 4 & D02250 & Octreotide acetate & hsa6751 & SSTR1 (Somatostatin Receptor 1) & $\begin{array}{l}\text { CTD: D015282 } \\
\text { PMID:16438887 }\end{array}$ \\
\hline 5 & D01712 & Theophylline sodium acetate & hsa136 & ADORA2B (adenosine A2b receptor) & KG: D01712 \\
\hline \multicolumn{6}{|c|}{ IC dataset (KEGG IDs) } \\
\hline 1 & D02098 & Proparacaine hydrochloride & hsa8645 & $\begin{array}{l}\text { KCNK5 (Potassium Two Pore Domain Channel Subfamily K } \\
\text { Member 5) }\end{array}$ & Unknown \\
\hline 2 & D00438 & Nimodipine & hsa779 & $\begin{array}{l}\text { CACNA1S (Calcium Voltage-Gated Channel Subunit } \\
\text { Alpha1S) }\end{array}$ & $\begin{array}{l}\text { KG: D00438 } \\
\text { DB:DB00393 }\end{array}$ \\
\hline 3 & D00649 & Amiloride hydrochloride & hsa8911 & CACNA1I (Calcium Voltage-Gated Channel Subunit Alpha1 I) & M: Amiloride (direct) \\
\hline 4 & D00495 & Paramethadione & hsa8913 & $\begin{array}{l}\text { CACNA1G (Voltage-dependent T-type calcium channel } \\
\text { subunit alpha-1G) }\end{array}$ & $\begin{array}{l}\text { KG: D00495 } \\
\text { DB:DB00617(indirect) }\end{array}$ \\
\hline 5 & D03365 & Nicotine & hsa1137 & CHRNA4 (Cholinergic Receptor Nicotinic Alpha 4 Subunit) & $\begin{array}{l}\text { PMID: } 17590520 \\
\text { KG: D03365 } \\
\text { DB: DB00184 }\end{array}$ \\
\hline \multicolumn{6}{|c|}{ Enzyme dataset (KEGG IDs) } \\
\hline 1 & D00542 & Halothane & hsa1571 & CYP2E1 (Cytochrome P450 2E1) & PMID:19442086 \\
\hline 2 & D00437 & Nifedipine & hsa1559 & CYP2C9 (Cytochrome P450 Family 2 Subfamily C Member 9) & $\begin{array}{l}\text { CTD: D009543 } \\
\text { PMID: } 9929518\end{array}$ \\
\hline 3 & D00139 & Methoxsalen & hsa1543 & CYP1A1 (Cytochrome P450 1A1) & PMID: 7702611 \\
\hline 4 & D00574 & Aminoglutethimide & hsa1589 & $\begin{array}{l}\text { CYP21A2 (Cytochrome P450 Family } 21 \text { Subfamily A Member } \\
\text { 2) }\end{array}$ & $\begin{array}{l}\text { M: Aminoglutethimide (indirect) } \\
\text { PMID: } 8201961\end{array}$ \\
\hline 5 & D00410 & Metyrapone & hsa1583 & $\begin{array}{l}\text { CYP11A1 (Cytochrome P450 Family } 11 \text { Subfamily A Member } \\
\text { 1) }\end{array}$ & CTD: D008797 \\
\hline \multicolumn{6}{|c|}{ FDA_DrugBank dataset (DrugBank IDs) } \\
\hline 1 & DB00801 & Halazepam & Q9UN88 & GABRQ (Gamma-aminobutyric acid receptor subunit theta) & $\begin{array}{l}\text { DB, UP, } \\
\text { C:3885575 } \\
\text { KG: D00338 }\end{array}$ \\
\hline 2 & DB01012 & Cinacalcet & 015530 & PDPK1 (3-phosphoinositide-dependent protein kinase 1) & Unknown \\
\hline 3 & DB00424 & Hyoscyamine & P08912 & CHRM5 (Muscarinic acetylcholine receptor M5) & KG: D00147- DG00053 \\
\hline 4 & DB01589 & Quazepam & P47870 & $\begin{array}{l}\text { GABRB2 (gamma-aminobutyric acid type A receptor subunit } \\
\text { beta2) }\end{array}$ & KG: D00457 \\
\hline 5 & DB00546 & Adinazolam & Q9UN88 & $\begin{array}{l}\text { GABRQ (gamma-aminobutyric acid type A receptor subunit } \\
\text { theta) }\end{array}$ & DB, KG: D02770 -DG00911 \\
\hline
\end{tabular}

C ChEMBL, CTD Comparative toxicogenomics database, DB DrugBank, M MATADOR, KG KEGG, DG chemical Drug Group from KEGG, PMID PubMed, UP UniProt Knowledgebase

dimensional space for each node. It directly applies the fusion function to create features for each drugtarget pair. On the other hand, DTiGEMS + calculated the cosine similarity for each drug-drug and target-target pair separately to formulate a new heterogeneous network, from which the path score features are obtained, resulting in losing some valuable features directly related to some drug-target edges. 
4. DTi2Vec FVs are the drug-target edge embeddings, while DTiGEMS + FVs are the sum and max pathscores for each drug-target edge under six path structures. This difference can lead to the best characteristic of DTi2Vec is that the FVs are not sparse contrary to the situation in DTiGEMS + , where the FVs can be sparse. The reason is that DTi2Vec leverages the node2vec embeddings to directly construct edge representation using fusion function, resulting in converting the sparse graph data into low dimensional vector spaces used as features. In contrast, DTiGEMS + used node2vec embeddings to calculate new cosine similarity and used these similarities to construct a new heterogeneous graph to obtain path score features. Although applying graph mining to extract path score features between each drug-target pair provides meaningful features for the classifier in DTiGEMS + , it can suffer from the sparsity issue, specifically when the number of known DTIs is limited, which affects the classifier's performance.

5. The last advantage of DTi2Vec over DTiGEMS is that DTi2Vec can handle the newDrug setting by predicting novel interactions for new drugs, and it achieved good results.

\section{Effectiveness of feature representation used with the heterogeneous network}

Most of the prior network-based methods have focused on a homogeneous network (by using separately the drug similarity graph and target similarity graph). Each node obtains the features from the corresponding subgraph and then predicts the edge connecting these two graphs by inferring DTI. Considering only the homogenous graph might result in losing some informative links and meaningful features that enhance the DTIs prediction accuracy. DTi2Vec takes advantage of combining two techniques of working on a complete heterogeneous DTIs graph with graph embedding (i.e., representation learning) that provides a powerful graph analytical approach to capture meaningful and rich features. Specifically, we constructed the full, rich, heterogeneous DTIs network augmented with KNN drug similarity and targets similarity graphs. We then apply the node2vec model to learn an embedding (i.e., feature representation) for each node (drug and target). These generated representations incorporate the node topological context and structural information so that similar nodes will have similar numerical representations. Node representations are learned via the Skip-gram model by optimizing the likelihood objective using stochastic gradient descent (SGD). Then, an efficient representation is generated for each drug-target edge using several fusion functions.

\section{Efficient performance in large scale data}

Many recent DTI prediction methods have performed well in terms of AUPR evaluation metric using Yamanishi_8 datasets, but none have achieved good AUPR using the approved FDA_DrugBank dataset. The possible reason for this may be because the previous methods did not consider the feature representation for drug-target edges (and did not distinguish between the positive and negative edges (i.e., unknown interaction)). DTi2Vec overcame this limitation and achieved improved predictive performance by obtaining high AUPR (0.88) in the FDA_DrugBank dataset.

\section{Effectiveness of the gradient boosting classifier}

Although DL using deep neural network (DNN) demonstrated its efficiency in prediction problems and outperformed most other ML classifiers, we believe that DNN doesn't perform well in the classification stage using the FVs of all drug-target pairs in our work. We tested our hypothesis by applying a sequential DL model in FVs for each dataset, and the results were less than the results obtained using XGBoost. The result suggests DNN works better dealing with unstructured data such as images, text (e.g., protein sequences or drug SMILES in DTIs prediction problem) and when using very large data, which is not the case for the Yamanishi_08 datasets. At the same time, ensemble-based algorithms (including boosting classifiers: AdaBoost and XGBoost) perform better in structured/tabular data. We selected XGBoost as the primary classifier in our work, a scalable and accurate gradient boosting machine. XGBoost classifier was developed and optimized for overall performance and computational speed. It has demonstrated the ability to push the boundaries of computing power for boosted trees algorithms. Furthermore, we compare XGBoost to several ML ensemble classifiers and the forward neural network classifier (FNN) but did not include the results in this manuscript since AdaBoost and XGBoost outperformed them all. Several of the previous DTIs prediction methods have applied different versions of the ensemble/boosting classifiers, but none of them leverage the advantage of using the XGBoost classifier. 


\section{Conclusion}

This work has developed an efficient computational method that solved DTI identification as a link prediction problem in heterogeneous networks. This method, DTi2Vec, takes advantage of the heterogeneous network to infer the interaction instead of separately using each homogeneous graph. It integrated the drug similarity graph, target similarity graph, and known DTIs graph to formulate the complete weighted heterogeneous graph $G(V, E)$, a more informative network to enhance the prediction performance. The Node2vec model was applied to this graph $G$, to generate efficient feature representations for each node and then learn feature representation for each drug-target edge using different fusion functions. These features proved to be very useful and informative, capturing all needed information from the graph for DTI prediction. It is generated automatically by omitting time-consuming feature engineering steps that can potentially affect prediction accuracy. We fed the FVs to two ensemble classifiers for a better comparison of prediction performance. DTi2Vec significantly increases the prediction performance compared to five state-of-the-art methods using Yamanishi_08 and FDA_DrugBank datasets and multiple evaluation metrics, which indicates the robustness of DTi2Vec. Furthermore, DTi2Vec demonstrates its efficiency in the reliability of results (based on the AUPR), and predicting new DTIs, validated using several databases and published literature.

One limitation worth mentioning is that DTi2Vec cannot predict the interaction of new targets or newDrugnewTarget pairs. We intend to overcome this limitation in future work by extending DTi2Vec capabilities to deal with other settings, including new target settings or newDrug-newTarget pairs, beyond the commonly applied random setting. As future work, we also plan to further improve the performance by applying and utilizing different representation learning algorithms (i.e., graph embedding techniques) and using different types of similarity of drug-drug and target-target that may be more informative. We also want to use our model to develop a real-life case study related to drug repositioning in cancer and experimentally validate our model's selected predictions to demonstrate our results' clinical relevance. Another extension of our method will be formulating the task as a regression model to predict the drugs and target binding ability that reflects more meaningful interaction. The last significant extension is that our network-based method is suitable for DTIs prediction, but it can be generalized to any network-based problem in biomedical domains such as drug-drug interactions network, drugdisease indication network, protein-disease association network, and others.

\section{Abbreviations}

Adaboost: Adaptive boosting; AUC: Area under ROC curve; AUPR: Area under the precision-recall curve; CART: Classification and Regression Trees; CV: Cross validation; DD sim: Drug-drug similarity matrix; DL: Deep learning; DTIs: Drugtarget interactions; E: Enzyme; ER: Error rate; FDA: Food and Drug Administration; FV: Feature vectors; FPR: False positive rate; GPCR: G-protein-coupled receptor; IC: Ion channel; KG: Knowledge graph; KNN: K-nearest neighbor; MAP: Mean average precision; MF: Matrix factorization; ML: Machine learning; MLP: Multilayer Perceptron; NBI: Network-based inference; NLP: Natural language processing; NN: Neural network; NR: Nuclear receptor; PSM: Path score model; P-value: Probability value; ROC: Receiver operating characteristic; TPR: True positive rate; TT sim: Target-target similarity matrix; XGBoost: EXtreme Gradient Boosting.

\section{Supplementary Information}

The online version contains supplementary material available at https://doi. org/10.1186/s13321-021-00552-w.

Additional file 1: Table S1. Tested Values (recommended by node2vec [1]) and the optimal parameter values for each dataset. Table S2. The AUC scores for all methods for each dataset separately. All results are rounded to 2 digits. Bold fonts with underline indicate the best results while bold fonts indicate the second-best. Table S3. DTi2Vec best performance for each dataset in terms of AUPR, in each fold of 10-folds CV, AvgAUPR, standard deviation (std), and p-values. Table S4. Performance of DTi2Vec in terms of AUPR using XGBoost classifier on each dataset with multiple FVs generated by applying different edge representation functions for new drug setting. Bold underlined font indicates the best result in each dataset.

\section{Acknowledgements}

The research reported in this publication was supported by the King Abdullah University of Science and Technology (KAUST).

\section{Authors' contributions}

MAT, VBB, and ME made the conception and designed the study. MAT did the implementation, and wrote the manuscript. RSO collected and organized FDA_DrugBank, performed several statistical analyses, and contributed to the result section. SA contributes to part of the first draft of the manuscript. MAT and SA validated and analyzed the novel interactions. MAT, XG, ME, SA, and $\mathrm{RSO}$ contributed to the discussions. ME, XG, and TG revised/edited the manuscript. All authors read and approved the final manuscript.

\section{Funding}

The research reported in this publication was supported by King Abdullah University of Science and Technology (KAUST) through the Awards Nos. BAS/1/1606-01-01, BAS/1/1059-01-01, BAS/1/1624-01-01, FCC/1/1976-20-01, and FCC/1/1976-26-01.

\section{Availability of data and materials}

All datasets and the source code used in this study are available in the following GitHub link: https://github.com/MahaThafar/DTi2Vec.

\section{Declarations}

\section{Competing interests}

The authors have declared that no conflict of interests exists.

\section{Author details}

${ }^{1}$ Computer, Electrical and Mathematical Sciences and Engineering Division (CEMSE), Computational Bioscience Research Center, Computer (CBRC), King Abdullah University of Science and Technology (KAUST), Thuwal, Kingdom of Saudi Arabia. ${ }^{2}$ College of Computers and Information Technology, Computer Science Department, Taif University, Taif, Kingdom of Saudi Arabia. ${ }^{3}$ The Jackson Laboratory for Genomic Medicine, Farmington, CT, USA. ${ }^{4}$ Faculty of Computing and Information Technology, King Abdulaziz University, Jeddah, Kingdom of Saudi Arabia. 
Received: 24 December 2020 Accepted: 5 September 2021

Published online: 22 September 2021

\section{References}

1. Jarada TN, Rokne JG, Alhajj R (2020) A review of computational drug repositioning: strategies, approaches, opportunities, challenges, and directions. J Cheminform 12(1):46

2. Masoudi-Nejad A, Mousavian Z, Bozorgmehr JH (2013) Drug-target and disease networks: polypharmacology in the post-genomic era. Silico Pharmacol 1:17

3. Chen X et al (2015) Drug-target interaction prediction: databases, web servers and computational models. Brief Bioinform 17(4):696-712

4. Ezzat A et al (2019) Computational prediction of drug-target interactions using chemogenomic approaches: an empirical survey. Brief Bioinform 20(4):1337-1357

5. Thafar M et al (2019) Comparison study of computational prediction tools for drug-target binding affinities. Front Chem 7:782

6. Dai $\mathrm{H}$ et al (2017) Sequence2Vec: a novel embedding approach for modeling transcription factor binding affinity landscape. Bioinformatics 33(22):3575-3583

7. Lagassé HAD et al (2017) Recent advances in (therapeutic protein) drug development. F1000Research 6:113

8. Sachdev K, Gupta MK (2019) A comprehensive review of feature based methods for drug target interaction prediction. J Biomed Inform 93:103159

9. Wu Z et al (2018) Network-based methods for prediction of drug-target interactions. Front Pharmacol 9:1134

10. Nanor E et al (2020) Featurization of drug compounds and target proteins for drug-target interaction prediction. Int J Sci Res Publ 10(2):9813

11. Zhang W et al (2019) Recent advances in the machine learning-based drug-target interaction prediction. Curr Drug Metab 20(3):194-202

12. Nath A, Kumari P, Chaube R (2018) Prediction of human drug targets and their interactions using machine learning methods: current and future perspectives. Methods Mol Biol 1762:21-30

13. Alonso H, Bliznyuk AA, Gready JE (2006) Combining docking and molecular dynamic simulations in drug design. Med Res Rev 26(5):531-568

14. Cummings M, Maxwell A, DesJarlais R (2007) Processing of small molecule databases for automated docking. Med Chem 3(1):107-113

15. Ma D-L, Chan DS-H, Leung C-H (2013) Drug repositioning by structurebased virtual screening. Chem Soc Rev 42(5):2130-2141

16. Qu K, Brooijmans N. Structure-based drug design. In: Computational methods for protein structure prediction and modeling; 2007, p. 135-176.

17. Lam JH et al (2019) A deep learning framework to predict binding preference of RNA constituents on protein surface. Nat Commun 10(1):4941

18. Mousavian Z, Masoudi-Nejad A (2014) Drug-target interaction prediction via chemogenomic space: learning-based methods. Expert Opin Drug Metab Toxicol 10(9):1273-1287

19. Rayhan F et al (2017) iDTI-ESBoost: identification of drug target interaction using evolutionary and structural features with boosting. Sci Rep 7(1):17731

20. Ding $\mathrm{H}$ et al (2014) Similarity-based machine learning methods for predicting drug-target interactions: a brief review. Brief Bioinform 15(5):734-747

21. Wang C, Kurgan L (2020) Survey of similarity-based prediction of drugprotein interactions. Curr Med Chem 27(35):5856-5886

22. Li H et al (2020) Modern deep learning in bioinformatics. J Mol $12: 823-827$

23. Li Y et al (2019) Deep learning in bioinformatics: Introduction, application, and perspective in the big data era. Methods 166:4-21

24. Lee I, Keum J, Nam H (2019) DeepConv-DTI: prediction of drug-target interactions via deep learning with convolution on protein sequences. PLoS Comput Biol 15(6):e1007129

25. Ba-Alawi W et al (2016) DASPfind: new efficient method to predict drugtarget interactions. J Cheminform 8:15

26. Zong N, Wong RSN, Ngo V (2019) Tripartite network-based repurposing method using deep learning to compute similarities for drug-target prediction. Methods Mol Biol 1903:317-328
27. Olayan RS, Ashoor H, Bajic VB (2018) DDR: efficient computational method to predict drug-target interactions using graph mining and machine learning approaches. Bioinformatics 34(7):1164-1173

28. Thafar MA et al (2020) DTiGEMS+: drug-target interaction prediction using graph embedding, graph mining, and similarity-based techniques. J Cheminformatics 12(1):44

29. Thafar MA et al. Computational drug-target interaction prediction based on graph embedding and graph mining. In: Proceedings of the 2020 10th international conference on bioscience, biochemistry and bioinformatics. 2020, Association for Computing Machinery. p. 14-21.

30. Bleakley K, Yamanishi Y (2009) Supervised prediction of drug-target interactions using bipartite local models. Bioinformatics 25(18):2397-2403

31. Buza K, Peška L (2017) Drug-target interaction prediction with Bipartite Local Models and hubness-aware regression. Neurocomputing 260:284-293

32. Cheng F et al (2012) Prediction of chemical-protein interactions network with weighted network-based inference method. PLoS ONE 7(7):e41064

33. Wu Z et al (2016) In silico prediction of chemical mechanism of action via an improved network-based inference method. Br J Pharmacol 173(23):3372-3385

34. Xuan P et al (2019) Gradient boosting decision tree-based method for predicting interactions between target genes and drugs. Front Genet 10:459

35. Alshahrani M et al (2017) Neuro-symbolic representation learning on biological knowledge graphs. Bioinformatics 33(17):2723-2730

36. Luo Y et al (2017) A network integration approach for drug-target interaction prediction and computational drug repositioning from heterogeneous information. Nat Commun 8(1):573

37. Mohamed SK, Nováček V, Nounu A (2019) Discovering protein drug targets using knowledge graph embeddings. Bioinformatics 36:603-610

38. Zeng $X$ et al (2020) Network-based prediction of drug-target interactions using an arbitrary-order proximity embedded deep forest. Bioinformatics 36(9):2805-2812

39. Alshahrani M, Thafar MA, Essack M (2021) Application and evaluation of knowledge graph embeddings in biomedical data. PeerJ Comput Sci 7:e341

40. Chen X, Liu MX, Yan GY (2012) Drug-target interaction prediction by random walk on the heterogeneous network. Mol Biosyst 8:1970-1978

41. Zheng $X$ et al. Collaborative matrix factorization with multiple similarities for predicting drug-target interactions. In: Proceedings of the 19th ACM SIGKDD international conference on Knowledge discovery and data mining. 2013, Association for Computing Machinery, p. 1025-1033.

42. Rifaioglu AS et al (2020) DEEPScreen: high performance drug-target interaction prediction with convolutional neural networks using 2-D structural compound representations. Chem Sci 11(9):2531-2557

43. Hao M, Bryant SH, Wang Y (2017) Predicting drug-target interactions by dual-network integrated logistic matrix factorization. Sci Rep 7:40376

44. Wang B et al (2014) Similarity network fusion for aggregating data types on a genomic scale. Nat Methods 11(3):333-337

45. Liu Y et al (2016) Neighborhood regularized logistic matrix factorization for drug-target interaction prediction. PLoS Comput Biol 12(2):e1004760

46. Yamanishi Y et al (2008) Prediction of drug-target interaction networks from the integration of chemical and genomic spaces. Bioinformatics 24:i232-i240

47. Kanehisa M et al (2017) KEGG: new perspectives on genomes, pathways, diseases and drugs. Nucleic Acids Res 45(D1):D353-D361

48. Kanehisa M et al (2006) From genomics to chemical genomics: new developments in KEGG. Nucleic Acids Res 34(Database issue):D354-D357

49. Schomburg I (2004) BRENDA, the enzyme database: updates and major new developments. Nucleic Acids Res 32(90001):431D - 433

50. Günther S et al (2008) SuperTarget and Matador: resources for exploring drug-target relationships. Nucleic Acids Res 36(Database issue):D919-D922

51. Wishart DS et al (2008) DrugBank: a knowledgebase for drugs, drug actions and drug targets. Nucleic Acids Res 36(Database issue):D901-D906 
52. Hattori Met al (2010) SIMCOMP/SUBCOMP: chemical structure search servers for network analyses. Nucleic Acids Res 38(Web Server issue):W652-W656

53. Smith SB et al (2012) Identification of common biological pathways and drug targets across multiple respiratory viruses based on human host gene expression analysis. PLoS ONE 7(3):e33174

54. Yue X et al (2020) Graph embedding on biomedical networks: methods, applications and evaluations. Bioinformatics 36(4):1241-1251

55. Su C et al (2020) Network embedding in biomedical data science. Brief Bioinform 21(1):182-197

56. Grover A, Leskovec J (2016) node2vec: scalable feature learning for networks. KDD 2016:855-864

57. Perozzi B, Al-Rfou R, Skiena S. DeepWalk: online learning of social representations. In: Proceedings of the 20th ACM SIGKDD international conference on knowledge discovery and data mining. 2014, ACM. p. 701-710.

58. Mikolov T et al (2013) Distributed representations of words and phrases and their compositionality. In: Burges CJC et al (eds) Advances in neural information processing systems. Curran Associates Inc, Red Hook, pp 3111-3119

59. Al Shalabi L, Shaaban Z. Normalization as a preprocessing engine for data mining and the approach of preference matrix. In: 2006 international conference on dependability of computer systems; 2006, p. 207-214

60. Liu A, Ghosh J, Martin CE. Generative oversampling for mining imbalanced datasets. In: DMIN. 2007. p. 66-72.

61. Lemaître G, Nogueira F, Aridas CK (2017) Imbalanced-learn: a python toolbox to tackle the curse of imbalanced datasets in machine learning. J Mach Learn Res 18(1):559-563

62. Friedman JH (2001) Greedy function approximation: a gradient boosting machine. Ann Stat 29(5):1189-1232

63. Garreta R, Moncecchi G. Learning scikit-learn: machine learning in Python. Packt Publishing Ltd; 2013, p. 100.

64. Chen T, Guestrin C. XGBoost: a scalable tree boosting system ACM SIGKDD international conference on knowledge discovery and data mining. ACM; 2016: p. 785-794
65. Davis J, Goadrich M. The relationship between precision-recall and ROC curves. In: Proceedings of the 23rd international conference on machine learning; 2006, ACM. p. 233-240.

66. Powers DM. Evaluation: from precision, recall and F-measure to ROC, informedness, markedness and correlation; 2011.

67. Wilcoxon F, Katti SK, Wilcox RA (1970) Critical values and probability levels for the Wilcoxon rank sum test and the Wilcoxon signed rank test. Select Tables Math Stat 1:171-259

68. Taheri SM, Hesamian G (2013) A generalization of the Wilcoxon signedrank test and its applications. Statist Papers 54(2):457-470

69. Robertson S. A new interpretation of average precision. In: Proceedings of the 31 st annual international ACM SIGIR conference on research and development in information retrieval. 2008, Association for Computing Machinery. p. 689-690.

70. Wishart DS et al (2018) DrugBank 5.0: a major update to the DrugBank database for 2018. Nucleic Acids Res 46(D1):D1074-D1082

71. Gaulton A et al (2017) The ChEMBL database in 2017. Nucleic Acids Res 45(D1):D945-D954

72. Kim S et al (2016) PubChem substance and compound databases. Nucleic Acids Res 44(D1):D1202-D1213

73. Davis AP et al (2017) The comparative toxicogenomics database: update 2017. Nucleic Acids Res 45(D1):D972-D978

74. Magrane M, UniProt C (2011) UniProt Knowledgebase: a hub of integrated protein data. Database 2011:bar009

\section{Publisher's Note}

Springer Nature remains neutral with regard to jurisdictional claims in published maps and institutional affiliations.
Ready to submit your research? Choose BMC and benefit from:

- fast, convenient online submission

- thorough peer review by experienced researchers in your field

- rapid publication on acceptance

- support for research data, including large and complex data types

- gold Open Access which fosters wider collaboration and increased citations

- maximum visibility for your research: over $100 \mathrm{M}$ website views per year

At $\mathrm{BMC}$, research is always in progress.

Learn more biomedcentral.com/submissions 